\title{
Vacunas contra Leishmania
}

\author{
Elisa Martínez-Silva', Yuritmia Ruiz de Ramos', Gilberto Bastidas-Pacheco²
}

\section{RESUMEN}

La leishmaniosis, una enfermedad parasitaria de amplia distribución mundial y endémica en muchas regiones, plantea un gran reto al control epidemiológico debido, por un lado, a la toxicidad cada vez mayor de la quimioterapia y a la resistencia a la misma, y por el otro, a que el control del vector se ha tornado extremadamente difícil porque el insecto se ha generalizado y adaptado a múltiples y diferentes microambientes; de allí que se le dé alta prioridad al desarrollo de estrategias profilácticas costo-efectivas para prevenirla, porque la población más afectada vive en países con economías débiles. El objetivo de esta revisión fue actualizar lo hecho en estrategias de vacunación contra Leishmania. Se resume dicha información para guiar a los profesionales de la salud sobre el adecuado proceder en el control de tan importante endemia mundial.

\section{PALABRAS CLAVE}

Leishmania; Leishmaniosis; Parásito; Profilaxis; Vacuna

\section{SUMMARY}

\section{Vaccines against Leishmania}

Leishmaniosis, a parasitic disease of worldwide distribution and endemic in many regions, poses a challenge to epidemiological control due, on the one hand, to the increased toxicity of chemotherapy and resistance to it and, on the other, to the fact that vector control has become extremely difficult because the insect is widespread and has adapted to many different microenvironments. Hence, the high priority given to develop prophylactic measures to prevent this disease, that are cost-effective because the affected population lives mostly in countries with weak economies. The subject of this review was to update what is being done in vaccination strategies against Leishmania. We summarize the information in order to guide health professionals in the adequate control of this important worldwide endemic disease.

Licenciada en Bioanálisis, Escuela de Bioanálisis, Facultad de Ciencias de la Salud, Universidad de Carabobo, Venezuela.

Médico Cirujano, MSc en Gerencia de la Educación, MSc en Protozoología, Doctor en Parasitología, Departamento de Salud Pública, Facultad de Ciencias de la Salud, Universidad de Carabobo, Venezuela.

Correspondencia: Gilberto Bastidas-Pacheco; bastidasprotozoo@hotmail.com

Recibido: julio 10 de 2015

Aceptado: agosto 5 de 2015

Cómo citar: Martínez-Silva E, Ruiz de Ramos Y, Bastidas-Pacheco G. Vacunas contra Leishmania. latreia. 2016 Abr-Jun;29(2):170-181. D0I 10.17533/udea.iatreia.v29n2a06 


\section{KEYWORDS}

Leishmania; Leishmaniosis, Parasite; Prophylaxis; Vaccine

\section{RESUM0}

\section{As vacinas contra Leishmania}

A leishmaniose, uma doença parasitária amplamente espalhada por todo o mundo, e endêmica em várias regiões, representa um grande desafio para o controle epidemiológico devido, por um lado, ao aumento da toxicidade da quimioterapia e a resistência ao mesmo, e por outro lado, para que o controle do vetor tornou-se extremamente difícil, porque o inseto tem sido espalhado e adaptado a diversas condições ambientais; dai que se dá alta prioridade para o desenvolvimento de estratégias de profilática eficaz em termos de custos para evitar, pois as pessoas mais afetadas vivem em países com economias frágeis. O objetivo da revisão foi o de atualizar o fato em estratégias de vacinação contra Leishmania. Se Resume essa informação para orientar os profissionais de saúde sobre o procedimento adequado para o controle de uma tão importante endemia mundial.

\section{PALAVRAS CHAVE}

Leishmania; Leishmaniose; Parasita; Profilaxia; Vacina

\section{GENERALIDADES}

La leishmaniosis es una enfermedad causada por protozoos del género Leishmania. Se trata de un parásito intracelular obligado que reside dentro del macrófago, donde prolifera, y que es transmitido por flebótomos. Se ha descrito la leishmaniosis en 98 países de cinco continentes, afecta a más de 12 millones de personas y cerca de 350 millones están en riesgo de padecerla. Cada año se producen entre 1,5 y 2 millones de casos nuevos de leishmaniosis tegumentaria y 500.000 de la forma visceral (1-3).

La leishmaniosis tegumentaria está presente en América Central y Suramérica, con excepción de Chile y Uruguay, y la forma visceral, en doce de sus países, aunque con baja frecuencia en los considerados andinos (Bolivia, Colombia, Ecuador, Perú y Venezuela), excepto Brasil que tiene morbimortalidad importante por leishmaniosis en alounas de sus regiones. La compleja distribución geográfica de la leishmaniosis en América obedece a variaciones en los ciclos de transmisión, los reservorios y los insectos transmisores, así como a los deficientes programas de control. Todo ello explica la tendencia al aumento de las personas afectadas y del riesgo de transmisión (4-6).

En la leishmaniosis, como en muchas de las enfermedades parasitarias, $y$ a pesar de las variadas estrategias empleadas por el parásito para sobrevivir en el mamífero, el hospedero puede adquirir inmunidad a la infección por la misma especie que agrupa al patógeno, a pesar de la respuesta inmune celular ineficiente dependiente de las citocinas y la polarización de los macrófagos. Por tanto, la vacunación podría ser exitosa para prevenir $y$ tratar la enfermedad $(1-3,7)$. $\mathrm{Tal}$ estrategia sería importante por la toxicidad de la quimioterapia y la resistencia a la misma, que cada vez son mayores, al igual que por otras circunstancias: aumento de la incidencia de la enfermedad en sujetos inmunocomprometidos, dificultad para el control epidemiológico y definición incompleta de los determinantes de susceptibilidad y progreso de la enfermedad (8).

\section{PRIMERA GENERACIÓN DE VACUNAS}

Se han desarrollado varias generaciones de vacunas contra la leishmaniosis: la primera se centró en parásitos muertos o extractos crudos $(9,10)$. Las vacunas fabricadas con parásitos muertos con o sin adyurante tienen las siguientes características: composición bioquímica estable, antigenicidad y tolerancia buenas, seguridad y bajo costo; fueron desarrolladas a partir de 1930 en Brasil y se han usado en Suramérica desde la década de 1940 (11), por ejemplo, en Colombia y Ecuador (12).

Estas vacunas han sido preparadas con Leishmania amazonensis y/o Leishmania major, lisadas por autoclave y con adyuvante de Freund completo $(13,14)$, o mediante la mezcla de promastigotos de Leishmania mexicana sometidos al autoclave y BCG (Bacilo de CaImette-Guérin). Se han usado para inmunoterapia o inmunoquimioterapia, que resultó efectiva contra la enfermedad cutánea leve y en la prevención de las lesiones cutáneas deformantes, pero no contra la 
leishmaniosis visceral $(10,12,15,16)$. En 1980 la vacunación contra la leishmaniosis con parásitos muertos experimentó un auge con base en la demostración de la excelente protección que confiere en ratones, cuando se administra intravenosa o intraperitonealmente; infortunadamente, con el tiempo los parásitos sometidos al autoclave pierden su potencia inmunogénica (17).

En el caso de la leishmaniosis visceral canina, se han empleado con éxito vacunas con fracciones del parásito, entre ellas la Leishmune ${ }^{\circledR}$, que consiste en una preparación enriquecida de glicoproteína de promastigotos de Leishmania donovani, llamada ligando de fucosa-manosa (FML, por su siogla en inglés) que es formulada con saponina de Quillaja saponania, como adyurante. La glicoproteína, el FML, es una molécula esencial (involucrada en la construcción del DNA del parásito) y constituye el mayor complejo antigénico de L. donovani (18). También se ha usado ampliamente como candidato para vacuna el lipofosfoglicano (LPG) de Leishmania spp., un ligando para TLR2 en células NK que regula la producción de IFN- $\gamma$ y TNF- $\alpha$, pero con resultados contradictorios (19).

Otro enfoque de la inmunoterapia consiste en usar una sola proteína antigénica del patógeno con el fin de provocar una respuesta inmunitaria específica; en este sentido, se ha empleado el péptido gp63 de Leishmania (leishmanolisina) por ser una proteína inmunógena en todas las especies de Leishmania. Esto lo hace un buen candidato para vacunas de péptidos contra este grupo de parásitos $(20,21)$.

También se han usado en perros vacunas contra Leishmania infantum atenuadas con gentamicina, aminoglucósido que afecta el metabolismo tiol-redox del parásito, que es crucial para su supervivencia cuando se expone al estrés oxidativo generado durante su entrada al macrófago del mamífero; el parásito puede penetrar, pero no sobrevive y genera una fuerte respuesta inmunitaria del hospedero (22).

Asimismo, en perros se demostró protección contra $L$. infantum cuando se los inmunizaba con antígenos de secreción/excreción purificados de sobrenadantes de cultivos de promastigotos de esa especie del parásito sin adyuvante (LiESAp) o con el adyuvante dipéptido muramil (LiESAp-MDP), lo que induce la proliferación de linfocitos específicos Th1, productores de IFN- $\gamma$, que potencian la actividad antileishmania mediada por el óxido nítrico secretado por monocitos derivados de macrófagos (23). Igual papel en la activación de la respuesta Th1 tienen las enzimas enolasa (LdEno) y aldolasa (LdAId) de la vía glicolítica extraídas de lisados solubles de aislamientos de L. donovani, y las serina-proteasas extracelulares de $L$. amazonensis, que incluso administrada por vía nasal confiere protección en ratones BALB/C (24). El mismo efecto lo pueden tener las siguientes proteínas solubles: F14, elF2, P45, PDI, TPI y TPR (25). De igual forma se recurre a la cisteína-proteasa en liposomas catiónicos con receptores agonistas contra $L$. donovani en el modelo del hámster $(26,27)$.

De la misma manera, pero en ratones, se ha empleado la vacunación con proteína ribosomal purificada de extractos crudos de promastigotos (LRP, por la sigla en inglés de Leishmania major ribosomal protein) combinada con el oligodeoxinucleótido CpG (CpG ODN), como adyurante, pues induce respuesta Th1 (28). Igualmente, se han usado antígenos de Leishmania spp., tales como: tiol antioxidante específico (TSA, por la sigla en inglés de thiol specific antioxidant), proteína 1 inductora de estrés (LmSTI1, por la siogla en inglés), cisteína peptidasa (CPA), histona $\mathrm{H} 1$, factor iniciador eucariótico de Leishmania (LelF), proteína 11 de membrana de quinetoplasto (KMP11, por la sigla en inglés), proteínas de membranas Dp 7 , gp 70-2 y FML, proteína B de superficie hidrófila acilada (HASPB, por la sigla en inglés), proteína específica de amastigoto (A2) y cisteína-proteinasa B (CPB, por la sigla en inglés), todos con efecto protector individual para solo una especie de Leishmania (25,29-39).

La inmunización con la enzima esterol metiltransferasa-c-24 (SMT, por la sigla en inglés) (formulada con una emulsión estable de monofosforil lípido A) es capaz de generar protección cruzada contra diferentes especies de Leishmania por ser una proteína altamente conservada entre ellas (36); esta proteína se requiere para la síntesis de ergosterol, el principal esterol de la membrana de Leishmania, que está ausente en los mamíferos. Con poca efectividad profiláctica se han empleado poliproteínas como Q, Leish-111f, Leish$110 f$ y KSAC (25).

Otra estrategia de vacunación es el uso de las proteínas de choque térmico (HSP, por la siogla en inglés) llamadas chaperonas moleculares porque intervienen en el 
plegado, montaje, localización intracelular, secreción y degradación de proteínas como las inmunoglobulinas y el complejo mayor de histocompatibilidad), debido a que son altamente conservadas y tienen potencial para estimular las células T. Al respecto, se ha usado satisfactoriamente la proteína de choque térmico 70 (HSP70) de L. donovani en el control de la infección en hámsteres $(40,41)$, incluso las observaciones indican que vacunas basadas en la combinación de HSP70 con proteínas estimuladoras Th1 (triosa fosfato isomerasa, proteína disulfuro-isomerasa y el factor 2 de elongación) de L. donovani potencian la efectividad en la destrucción del parásito intracelular (42).

\section{SEGUNDA GENERACIÓN DE VACUNAS}

Se basa en una antigua observación: que después de curarse de una lesión el individuo se hace resistente a la reinfección. Esta práctica, conocida como Ieishmanización, consiste en la inoculación deliberada de formas vivas de Leishmania, procedentes del exudado de lesiones cutáneas de personas infectadas, en sitios anatómicos no expuestos de individuos sanos. Esta técnica ha sido practicada por siglos $(43,44)$ y a gran escala en Israel, Irán y la extinta Unión Soviética $(11,17)$. Sin embargo, su empleo se ha limitado por razones éticas y por la aparición de efectos adversos, como el desarrollo de lesiones persistentes, psoriasis, inmunosupresión, hipersensibilidad y persistencia del parásito; también por las dificultades en la cuantificación del inóculo $(9,11,17,45)$.

La siguiente vía en el desarrollo de vacunas contra Leishmania fue el uso de parásitos vivos atenuados por medios físicos, químicos o genéticos, a saber: cultivos in vitro por largos períodos, sensibilidad a la temperatura, atenuación por rayos gamma, atenuación química, mutagénesis química, cultivos bajo presión de drogas y modificaciones del parásito como la disrupción genética (46-50). Sin embargo, los principales problemas del uso de parásitos atenuados son la seguridad y la poca factibilidad de utilizarlos a gran escala en el campo $(42,51)$.

Al respecto, la atenuación por modificación genética de los parásitos permite reducir la virulencia, mantener la inmunogenicidad $y$ producir una respuesta inmunitaria similar a la obtenida con los parásitos vivos y virulentos, pero sin el daño asociado a estos (11).
Con la secuenciación completa de L. major se puede atenuar su virulencia mediante el bloqueo o reemplazo (knock-out) de genes esenciales $(7,52)$. Lo que se pretende es diseñar parásitos sin los genes que les garantizan larga supervivencia dentro del hospedero, haciéndolos, por tanto, seguros para inmunizar (44). Por ejemplo, en L. major se modificó el gen de dihidrofolato reductasa-timidilato sintasa (DHFR-TS), y en L. mexicana la cisteína-proteasa CPA y CCPB (18,53-55). Asimismo, por deleciones se han desarrollado leishmanias knock-out para los genes A2 rel, SIR2 y Hsp70-II y para genes de proteínas esenciales como: cisteína-proteinasa, transportador de bioterina, complejo citocromo $C$ oxidasa, proteína parecida a ubiquitina, proteína que convierte el precursor Ufm1 a su forma conjugada y proteína p27 específica de amastiootos (26,54,56-63).

En la atenuación de la virulencia de Leishmania se ha recurrido a la adición de casetes suicidas que ocasionan la muerte del parásito en respuesta a un determinado estímulo externo, como la introducción de genes de sensibilidad a drogas (17), por ejemplo: la expresión en L. major de los genes timidina-quinasa del virus herpes tipo 1 que la hacen sensible al ganciclovir (64) y el gen de la citosina-deaminasa de Sacharomyces cerevisiae, que hace al parásito sensible a drogas como 5 -fluorocitosina (65).

Otro enfoque de las vacunas de segunda generación es el uso de virus o bacterias vivos recombinantes que expresan antígenos de Leishmania. Ambos, virus y bacterias, expresan proteínas del parásito y actúan como sistema coadyuvante. Son ejemplos de proteínas del parásito expresadas por bacterias con este propósito: la proteasa de superficie gp63 de L. major, el antígeno de superficie clonado en SaImonella thypymurium mutante (patógeno intracelular capaz de penetrar en el macrófago humano), sola o en asociación con IL-2, IFN- $\gamma$, TNF- $\alpha$ o antígeno LACK (66); o en BCG (67) o Lactococcus lactis (bacteria grampositiva no patógena, usada en la fermentación de alimentos) que expresan A2 de L. donovani y L. Iactis y coexpresan LACK e IL1249.

Los siguientes son ejemplos de virus que expresan proteínas vacunantes: a) virus de la vaccinia que expresa la proteína de superficie del promastigoto G46/M-2/ PSA-2, que protege contra $L$. amazonensis (68), análogo en el parásito del receptor para activar la quinasa $C$ de los mamíferos que protege a ratones BALB/C 
contra L major (69); b) adenovirus que expresan el antígeno A2 de amastigotos (70).

También se han empleado parásitos distintos a Leishmania en la técnica de vacunación con base en la atenuación como el antígeno LCR1 de L. chagasi (similar a una proteína flagelar de Tripanosoma cruzi) en BCG (71); el antígeno proteico de la membrana del quinetoplasto KMP-11 en taquizoítos atenuados de Toxoplasma gondii (72) y Leishmania tarentolae (no patógena para humanos), bien sea solo, recombinante con expresión única del gen A2 o fusionado con los genes CPA, CPB y CTE49. Este último enfoque para las vacunas vivas atenuadas, es decir, el empleo de Leishmanias no patógenas para el ser humano, fue ideado por Breton (73), con base en el alto nivel de inmunorreactividad cruzada que se observa entre especies de parásitos patógenos y no patógenos para el ser humano, y podría llegar a ser la vacuna que mayor seguridad ofrezca al receptor en esta categoría.

También es posible modificar los parásitos para que produzcan sustancias biológicas que activen el sistema inmunitario, como el factor estimulador de colonias de granulocitos y macrófagos (GM-CSF) (74). Sin embargo, hay varios argumentos en contra de las vacunas atenuadas, entre ellos, que la deleción de genes de virulencia esenciales pueda ocasionar la completa destrucción del parásito, o la aparición de mutantes que favorezcan el desarrollo de lesión (73).

Por esta razón, actualmente se está investigando con la radiación ionizante en la atenuación de Leishmania para el desarrollo de vacunas, empleando para ello el modelo del ratón $\mathrm{BALB} / \mathrm{c}$ y las vías de administración intramuscular e intraperitoneal $(49,75)$.

\section{TERCERA GENERACIÓN DE VACUNAS}

Incluye, como el procedimiento de vacunación más reciente, la codificación de genes para un antígeno de protección, clonado en un vector; en pocas palabras, este método implica la inoculación de DNA insertado en un plásmido bacteriano que actúa como vector. EI gen del antígeno vacunal queda bajo el control de un potente promotor mamífero. Cuando se inocula en un animal este plásmido, construido por ingeniería genética, es capturado por las células del hospedero, en las cuales el DNA se transcribe a RNAm y se traduce en la proteína endógena de la vacuna $(33,76,77)$.

De esta generación la sustancia más probada como candidato para vacuna recombinante es la proteína de superficie B1 acilada hidrófila (HASPB1), que confiere protección contra L. donovani (31). También se ha empleado el candidato antigénico NH36, que confiere protección contra la infección por L chagasi, L. mexicana (32) y L. amazonensis. Esto plantea su efectividad potencial como vacuna inmunoprofiláctica bivalente, para el control de ambas endemias. A la par se han empleado las proteínas ribosomales recombinantes L3 y L5 de L. major para el desarrollo de vacunas contra Leishmania (78). Asimismo, la vacuna de DNA basada en la expresión de la proteína antioxidante tiol específica de amastigoto y promastigoto induce respuesta inmune de la plataforma Th1, cuyo efecto puede amplificarse cuando se acompaña con aluminio: este es un ejemplo de los intentos por impulsar las vacunas DNA con adyuvantes $(79,80)$. Otros antígenos de Leishmania candidatos para vacuna de DNA son el factor de elongación e iniciación, K26/ HASPB, la quinasa $C$ activa, la nucleósido-hidrolasa, P36LACK, A2, gp63, KMP11, CPB, ORFF, UBQ-ORFF, TRYP, H2A, H2B, H3, H4, GCS- $\gamma$, PSA-2 y la esterol-metiltransferasa $24-C(25,42,81)$.

Coler y colaboradores (82) han trabajado en leishmaniosis visceral con tres poliproteínas recombinantes de Leishmania (TSA, LmSTI1, LeIF) en asociación con el lípido monofosforil y el hidrocarburo escualeno como coadyuvante (MPL-SE). En Europa se están haciendo pruebas clínicas de dos nuevas vacunas de DNA (una basada en un vector viral, y la otra, en la expresión genética de antígenos seleccionados de Leishmania denominada LEISHDNAVAX2) (51).

Por las siguientes razones son notorias las ventajas de estas estrategias profilácticas sobre las anteriores: son vacunas simples, se pueden producir a gran escala, son estables a temperatura ambiente, lo que facilita su almacenamiento $y$ transporte, $y$, finalmente, se pueden combinar varios antígenos $y$ administrarlos en una sola dosis y por tanto brindar protección contra más de una especie de Leishmania, con lo cual se reduce el número de vacunas (83).

A las categorías de vacunas ya mencionadas se suman otros enfoques: la disrupción, si se quiere química, de 
la señalización mediante el knock-out genético del factor de transcripción STAT6 para reducir la expresión de la enzima arginasa, molécula que contribuye a la replicación intracelular de Leishmania por competencia con la óxido nítrico sintetasa (NOS2) por el sustrato arginina y por la generación de poliaminas que promueven el crecimiento del parásito (84); las células $\mathrm{T}$ asesinas (únicas que reconocen glicolípidos) estimuladas con el antígeno alfa galactosil ceramida (aGalCer) para incrementar su efecto natural en la infección (85); el uso con fines de vacunación de células dendríticas pulsadas ex vivo con antígenos de Leishmania, pues actuarían como vectores de antígenos y adyurantes naturales, en la estimulación de respuestas protectoras, como lo demostraron Moll y Berberich (86), porque las células dendríticas son muy especializadas en la presentación de antígenos, mecanismo esencial para la generación de células T en la respuesta inmunitaria protectora, directamente dependiente de la naturaleza del estímulo microbiano. La manipulación de estas células para la generación de una respuesta celular Th1 específica de antígeno parece ser una estrategia promisoria de vacunación contra Leishmania. (87-90).

También se han empleado con éxito en vacunación e inmunoterapia en un modelo de ratón (91) células dendríticas que sobreexpresan IL-12 (citocina que estimula Ia respuesta inmunitaria), y fragmentos o exosomas derivados de células dendríticas previamente expuestas a lisados de Leishmania (92). Además, se han pulsado células dendríticas con péptidos específicos del parásito como gp63 o con péptidos multiepítope $(93,94)$. Asimismo, se han usado en las terapias con células dendríticas adyuvantes como el CpG-ODN, que es un ligando TLR9, que induce la activación de dichas célulasyestimula la respuesta Th 1 (95). In vivo, se ha manipulado el receptor DEC205 de las células dendríticas con antígenos (LACK, Lelf, LmSTI1a) conjugados con anticuerpos como herramienta de inmunización contra Leishmania (96). Finalmente, se ha usado la transfectación genética FIt3L in vivo con el objeto de incrementar la población de células dendríticas (97).

Por su efecto contra tripanosomas, se ha usado el extracto de Warburgia ugandensis subsp. ugandensis, planta de uso medicinal en África, con antígenos solubles de L. major como vacuna en ratones BALB/c (98), y las vacunas con componentes de la saliva del flebótomo, en particular los antígenos MAX o MAXIDILAN (99), PpSP15, LJM19, LJL143 y LJM17, proteínas obtenidas de este insecto que inducen resistencia significativa contra la infección por L. major y L. infantum (100). Actualmente se prueba el efecto protector de una vacuna basada en la proteína PpSp15 de la saliva del vector con Leishmania tarentolae viva recombinante que expresa proteína-cisteinasa (101). Para finalizar, es prudente reconocer que ninguna de las estrategias de vacunación desarrolladas hasta el momento es capaz de controlar eficientemente la enfermedad.

\section{REFERENCIAS BIBLIOGRÁFICAS}

1. Nylén S, Gautam S. Immunological perspectives of leishmaniasis. J Glob Infect Dis. 2010 May;2(2):13546. DOI 10.4103/0974-777X.62876.

2. Mougneau E, Bihl F, Glaichenhaus N. Cell biology and immunology of Leishmania. Immunol Rev. 2011 Mar;240(1):286-96. DOI 10.1111/j.1600065X.2010.00983.X.

3. Alvar J, Vélez ID, Bern C, Herrero M, Desjeux P, Cano $\mathrm{J}$, et al. Leishmaniasis worldwide and global estimates of its incidence. PLoS One. 2012;7(5):e35671. DOI 10.1371/journal.pone.0035671.

4. Organización Mundial de la Salud. Lucha contra la Leishmaniasis. Informe de un comité de expertos de Ia OMS. Ginebra: OMS; 1990. Serie de Informes Técnicos: 793.

5. Davies CR, Reithinger R, Campbell-Lendrum D, Feliciangeli D, Borges R, Rodriguez N. The epidemiology and control of leishmaniasis in Andean countries. Cad Saude Publica. 2000 Oct-Dec;16(4):925-50.

6. Organización Panamericana de la Salud. Segunda reunión de los programas nacionales de leishmaniasis en las Américas. Informe final. Ciudad de Panamá; 2013.

7. Coler RN, Reed SG. Second-generation vaccines against leishmaniasis. Trends Parasitol. 2005 May;21(5):244-9.

8. Palatnik-de-Sousa CB. Vaccines for leishmaniasis in the fore coming 25 years. Vaccine. 2008 Mar;26(14):1709-24. DOI 10.1016/j.vaccine.2008.01.023. 
9. Modabber F. Vaccines against leishmaniasis. Ann Trop Med Parasitol. 1995 Dec;89 Suppl 1:83-8.

10. Khamesipour A, Rafati S, Davoudi N, Maboudi F, Modabber F. Leishmaniasis vaccine candidates for development: a global overview. Indian J Med Res. 2006 Mar;123(3):423-38.

11. Handman E. Leishmaniasis: current status of vaccine development. Clin Microbiol Rev. 2001 Apr; 14(2):229-43.

12. Noazin S, Modabber F, Khamesipour A, Smith PG, Moulton LH, Nasseri K, et al. First generation leishmaniasis vaccines: a review of field efficacy trials. Vaccine. 2008 Dec;26(52):6759-67. DOI 10.1016/j.vaccine.2008.09.085.

13. Antunes CM, Mayrink W, Magalhaes PA, Costa CA, Melo MN, Dias M, et al. Controlled field trials of a vaccine against New World cutaneous leishmaniasis. Int J Epidemiol. 1986 Dec;15(4):572-80.

14. Armijos RX, Weigel MM, Calvopina M, Hidalogo A, Cevallos W, Correa J. Safety, immunogenecity, and efficacy of an autoclaved Leishmania amazonensis vaccine plus BCG adjuvant against New World cutaneous leishmaniasis. Vaccine. 2004 Mar;22(910):1320-6.

15. Cabrera M, Blackwell JM, Castes M, Trujillo D, Convit J, Shaw MA. Immunotherapy with live BCG plus heat killed Leishmania induces a $\mathrm{T}$ helper 1-like response in American cutaneous leishmaniasis patients. Parasite Immunol. 2000 Feb;22(2):73-9.

16. Convit J, Ulrich M, Polegre MA, Avila A, Rodríguez N, Mazzedo MI, et al. Therapy of Venezuelan patients with severe mucocutaneous or early lesions of diffuse cutaneous leishmaniasis with a vaccine containing pasteurized Leishmania promastigotes and bacillus Calmette-Guerin: preliminary report. Mem Inst OswaIdo Cruz. 2004 Feb;99(1):57-62.

17. Khamesipour A, Dowlati Y, Asilian A, HashemiFesharki R, Javadi A, Noazin S, et al. Leishmanization: use of an old method for evaluation of candidate vaccines against leishmaniasis. Vaccine. 2005 May;23(28):3642-8.

18. Santos WR, Aguiar IA, Paraguai de Souza E, de Lima VM, Palatnik M, Palatnik-de-Sousa CB. Immunotherapy against murine experimental visceral Ieishmaniasis with the FML-vaccine. Vaccine. 2003 Dec;21(32):4668-76.
19. Pinheiro RO, Pinto EF, Lopes JR, Guedes HL, Fentanes RF, Rossi-Bergmann B. TGF-beta-associated enhanced susceptibility to leishmaniasis following intramuscular vaccination of mice with Leishmania amazonensis antigens. Microbes Infect. 2005 Oct;7(13):1317-23.

20. Seay MB, Heard PL, Chaudhuri G. Surface Zn-proteinase as a molecule for defense of Leishmania mexicana amazonensis promastigotes against cytolysis inside macrophage phagolysosomes. Infect Immun. 1996 Dec;64(12):5129-37.

21. Rezvan H. Immunogenicity of HLA-DR1 Restricted Peptides Derived from Leishmania major gp63 Using FVB/N-DR1 Transgenic Mouse Model. Iran J Parasitol. 2013 Apr;8(2):273-9.

22. Daneshvar H, Namazi MJ, Kamiabi H, Burchmore R, Cleaveland S, Phillips S. Gentamicin-attenuated Leishmania infantum vaccine: protection of dogs against canine visceral leishmaniosis in endemic area of southeast of Iran. PLoS Neogl Trop Dis. 2014 Apr;8(4):e2757. DOI 10.1371/journal. pntd.0002757. Erratum in: PLoS Negl Trop Dis. 2015 May;9(5):e0003787.

23. Lemesre JL, Holzmuller P, Cavaleyra M, Gonçalves RB, Hottin G, Papierok G. Protection against experimental visceral leishmaniasis infection in dogs immunized with purified excreted secreted antigens of Leishmania infantum promastiogotes. Vaccine. 2005 Apr;23(22):2825-40.

24. Gupta R, Kumar V, Kushawaha PK, Tripathi CP, Joshi S, Sahasrabuddhe AA, et al. Characterization of glycolytic enzymes--rAldolase and rEnolase of Leishmania donovani, identified as Th1 stimulatory proteins, for their immunogenicity and immunoprophylactic efficacies against experimental visceral leishmaniasis. PLoS One. 2014 Jan;9(1):e86073. DOI 10.1371/journal.pone.0086073.

25. Joshi S, Rawat K, Yadav NK, Kumar V, Siddiqi MI, Dube A. Visceral Leishmaniasis: Advancements in Vaccine Development via Classical and Molecular Approaches. Front Immunol. 2014 Aug;5:380. DOI 10.3389/fimmu.2014.00380.

26. Das A, Ali N. Combining cationic liposomal delivery with MPL-TDM for cysteine protease cocktail vaccination against Leishmania donovani: evidence for antigen synergy and protection. PLoS Negl Trop Dis. 2014 Aug;8(8):e3091. DOI 10.1371/journal.pntd.0003091. 
27. de Matos Guedes HL, da Silva Costa BL, Chaves SP, de Oliveira Gomes DC, Nosanchuk JD, De Simone $S G$, et al. Intranasal vaccination with extracellular serine proteases of Leishmania amazonensis confers protective immunity to BALB/c mice against infection. Parasit Vectors. 2014 Sep; 7:448. DOI 10.1186/1756-3305-7-448.

28. Iborra S, Parody N, Abánades DR, Bonay P, Prates D, Novais FO, et al. Vaccination with the Leishmania major ribosomal proteins plus $\mathrm{CpG}$ oligodeoxynucleotides induces protection against experimental cutaneous leishmaniasis in mice. Microbes Infect. 2008 Aug-Sep;10(10-11):1133-41. DOI 10.1016/j.micinf.2008.06.002.

29. Wilson ME, Young BM, Andersen KP, Weinstock JV, Metwali A, Ali KM, et al. A recombinant Leishmania chagasi antigen that stimulates cellular immune responses in infected mice. Infect Immun. 1995 May;63(5):2062-9.

30. Webb JR, Campos-Neto A, Orendale PJ, Martin TI, Stromberg EJ, Badaro R, et al. Human and murine immune responses to a novel Leishmania major recombinant protein encoded by members of a multicopy gene family. Infect Immun. 1998 Jul;66(7):327989.

31. Stäger S, Smith DF, Kaye PM. Immunization with a recombinant stage-regulated surface protein from Leishmania donovani induces protection against visceral leishmaniasis. J Immunol. 2000 Dec;165(12):7064-71

32. Ghosh A, Zhang WW, Matlashewski G. Immunization with $\mathrm{A} 2$ protein results in a mixed Th1/Th2 and a humoral response which protects mice against Leishmania donovani infections. Vaccine. 2001 Oct;20(1-2):59-66.

33. Campos-Neto A, Porrozzi R, Greeson K, Coler RN, Webb JR, Seiky YA, et al. Protection against cutaneous leishmaniasis induced by recombinant antigens in murine and nonhuman primate models of the human disease. Infect Immun. 2001 Jun;69(6):4103-8.

34. Aguilar-Be I, da Silva Zardo R, Paraguai de Souza E, Borja-Cabrera GP, Rosado-Vallado M, Mut-Martin $M$, et al. Cross-protective efficacy of a prophylactic Leishmania donovani DNA vaccine against visceral and cutaneous murine leishmaniasis. Infect Immun. 2005 Feb;73(2):812-9.
35. Basu R, Bhaumik S, Basu JM, Naskar K, De T, Roy S. Kinetoplastid membrane protein-11 DNA vaccination induces complete protection against both pentavalent antimonial-sensitive and -resistant strains of Leishmania donovani that correlates with inducible nitric oxide synthase activity and IL-4 generation: evidence for mixed Th1- and Th2-like responses in Visceral Leishmaniasis. J Immunol. 2005 Jun; 174(11):7160-71. DOI 10.4049/jimmunol.174.11.7160.

36. Tewary P, Jain M, Sahani MH, Saxena S, Madhubala R. A heterologous prime-boost vaccination regimen using ORFF DNA and recombinant ORFF protein confers protective immunity against experimental visceral leishmaniasis. J Infect Dis. 2005 Jun;191(12):2130-7.

37. Rafati S, Zahedifard F, Nazgouee F. Prime-boost vaccination using cysteine proteinases type I and II of Leishmania infantum confers protective immunity in murine visceral leishmaniasis. Vaccine. 2006 Mar;24(12):2169-75.

38. Agallou M, Athanasiou E, Koutsoni O, Dotsika E, Karagouni E. Experimental Validation of Multi-Epitope peptides Including Promising MHC Class I- and IIRestricted Epitopes of Four Known Leishmania infantum Proteins. Front Immunol. 2014 Jun;5:268. DOI 10.3389/fimmu.2014.00268.

39. Goto Y, Bhatia A, Raman VS, Vidal SE, Bertholet S, Coler RN, et al. Leishmania infantum sterol 24-cmethyltransferase formulated with MPL-SE induces cross-protection against L. major infection. Vaccine. 2009 May;27(21):2884-90. DOI 10.1016/j.vaccine.2009.02.079.

40. Srivastava PK, Menoret A, Basu S, Binder RJ, McQuade KL. Heat shock proteins come of age: primitive functions acquire new roles in an adaptive world. Immunity. 1998 Jun;8(6):657-65.

41. Oladiran A, Belosevic M. Trypanosoma carassii hsp70 increases expression of inflammatory cytokines and chemokines in macrophages of the goldfish (Carassius auratus L.). Dev Comp Immunol. 2009 Oct;33(10):1128-36. DOI 10.1016/j.dci.2009.06.003.

42. Jaiswal AK, Khare P, Joshi S, Kushawaha PK, Sundar S, Dube A. Th1 stimulatory proteins of Leishmania donovani: comparative cellular and protective responses of rTriose phosphate isomerase, rProtein disulfide isomerase and rElongation factor-2 in 
combination with rHSP70 against visceral leishmaniasis. PLoS One. 2014 Sep;9(9):e108556. DOI 10.1371/journal.pone.0108556.

43. Handman E. Leishmania virulence: it's a knock out! Trends Parasitol. 2001 Feb;17(2):60.

44. Kedzierski L. Leishmaniasis Vaccine: Where are We Today? J Glob Infect Dis. 2010 May;2(2):177-85. DOI 10.4103/0974-777X.62881.

45. Reithinger R, Brooker S, Kolaczinski JH. Visceral leishmaniasis in eastern Africa--current status. Trans R Soc Trop Med Hyg. 2007 Dec;101(12):1169-70.

46. Mitchell GF, Handman E, Spithill TW. Vaccination against cutaneous leishmaniasis in mice using nonpathogenic cloned promastigotes of Leishmania major and importance of route of injection. Aust J Exp Biol Med Sci. 1984 Apr;62 (Pt 2):145-53.

47. Gorczynski RM. Immunization of susceptible $\mathrm{BALB} / \mathrm{c}$ mice against Leishmania braziliensis. II. Use of temperature-sensitive avirulent clones of parasite for vaccination purposes. Cell Immunol. 1985 Aug;94(1):11-20.

48. Kimsey PB, Theodos CM, Mitchen TK, Turco SJ, Titus RG. An avirulent lipophosphoglycan-deficient Leishmania major clone induces $\mathrm{CD} 4+\mathrm{T}$ cells which protect susceptible BALB/c mice against infection with virulent L. major. Infect Immun. 1993 Dec;61(12):5205-13.

49. Rivier D, Shah R, Bovay P, Mauel J. Vaccine development against cutaneous leishmaniasis. Subcutaneous administration of radio attenuated parasites pro- tects CBA mice against virulentLeishmania major challenge. Parasite Immunol. 1993 Feb; 15(2):7584. DOI 10.1111/j.1365-3024.1993.tb00587.x.

50. Daneshvar H, Coombs GH, Hagan P, Phillips RS. Leishmania mexicana and Leishmania major: attenuation of wild-type parasites and vaccination with the attenuated lines. J Infect Dis. 2003 May;187(10):1662-8.

51. Saljoughian N, Taheri T, Rafati S. Live vaccination tactics: possible approaches for controlling visceral leishmaniasis. Front Immunol. 2014 Mar;5:134. DOI 10.3389/fimmu.2014.00134.

52. Stober CB, Lange UG, Roberts MT, Gilmartin B, Francis R, Almeida R, et al. From genome to vaccines for leishmaniasis: screening 100 novel vaccine candidates against murine Leishmania major infection. Vaccine. 2006 Mar;24(14):2602-16.

53. Titus RG, Gueiros-Filho FJ, de Freitas LA, Beverley SM. Development of a safe live Leishmania vaccine line by gene replacement. Proc Natl Acad Sci U S A. 1995 Oct;92(22):10267-71.

54. Alexander J, Coombs GH, Mottram JC. Leishmania mexicana cysteine proteinase-deficient mutants have attenuated virulence for mice and potentiate a Th1 response. J Immunol. 1998 Dec;161(12): 6794-801.

55. Selvapandiyan A, Debrabant A, Duncan R, Muller J, Salotra P, Sreenivas G, et al. Centrin gene disruption impairs stage-specific basal body duplication and cell cycle progression in Leishmania. J Biol Chem. 2004 Jun;279(24):25703-10.

56. Souza AE, Bates PA, Coombs GH, Mottram JC. Null mutants for the Imcpa cysteine proteinase gene in Leishmania mexicana. Mol Biochem Parasitol. 1994 Feb;63(2):213-20.

57. Zhang WW, Matlashewski G. Characterization of the A2-A2rel gene cluster in Leishmania donovani: involvement of A2 in visceralization during infection. Mol Microbiol. 2001 Feb;39(4):935-48.

58. Papadopoulou B, Roy G, Breton M, Kündig C, Dumas C, Fillion I, et al. Reduced infectivity of a Leishmania donovani biopterin transporter genetic mutant and its use as an attenuated strain for vaccination. Infect Immun. 2002 Jan;70(1):62-8.

59. Silvestre R, Cordeiro-Da-Silva A, Santarém N, Vergnes B, Sereno D, Ouaissi A. SIR2-deficient Leishmania infantum induces a defined IFN-gamma/IL-10 pattern that correlates with protection. J Immunol. 2007 Sep;179(5):3161-70.

60. Selvapandiyan A, Dey R, Gannavaram S, LakhalNaouar I, Duncan R, Salotra P, et al. Immunity to visceral leishmaniasis using genetically defined liveattenuated parasites. J Trop Med. 2012;2012:631460. DOI 10.1155/2012/631460.

61. Dey R, Meneses C, Salotra P, Kamhawi S, Nakhasi HL, Duncan R. Characterization of a Leishmania stage-specific mitochondrial membrane protein that enhances the activity of cytochrome c oxidase and its role in virulence. Mol Microbiol. 2010 Jul;77(2):399-414. DOI 10.1111/j.13652958.2010.07214.x. 
62. Gannavaram S, Connelly PS, Daniels MP, Duncan R, Salotra P, Nakhasi HL. Deletion of mitochondrial associated ubiquitin fold modifier protein Ufm 1 in Leishmania donovani results in loss of $\beta$-oxidation of fatty acids and blocks cell division in the amastigote stage. Mol Microbiol. 2012 Oct;86(1):187-98. DOI 10.1111/j.1365-2958.2012.08183.x.

63. Gannavaram S, Davey S, Lakhal-Naouar I, Duncan R, Nakhasi HL. Deletion of ubiquitin fold modifier protein Ufm 1 processing peptidase Ufsp in L. donovani abolishes Ufm 1 processing and alters pathogenesis. PLoS Negl Trop Dis. 2014 Feb;8(2):e2707. DOI 10.1371/journal.pntd.0002707.

64. Muyombure A, Olivier M, Harvie P, Bergeron MG, Ouellette M, Papadopoulou B. Protection against Leishmania major challenge infection in mice vaccinated with live recombinant parasites expressing a cytotoxic gene. J Infect Dis. 1998 Jan;177(1):188-95.

65. Davoudi N, Tate CA, Warburton C, Murray A, Mahboudi F, McMaster WR. Development of a recombinant Leishmania major strain sensitive to ganciclovir and 5-fluorocytosine for use as a live vaccine challenge in clinical trials. Vaccine. 2005 Jan;23(9):1170-7.

66. Yang DM, Fairweather N, Button LL, McMaster WR, KahI LP, Liew FY. Oral Salmonella typhimurium (AroA-) vaccine expressing a major leishmanial surface protein (gp63) preferentially induces T helper 1 cells and protective immunity against leishmaniasis. J Immunol. 1990 Oct;145(7):2281-5.

67. Connell ND, Medina-Acosta E, McMaster WR, Bloom BR, Russell DG. Effective immunization against cutaneous leishmaniasis with recombinant bacilli Calmette-Guérin expressing the Leishmania surface proteinase gp63. Proc Natl Acad Sci U S A. 1993 Dec;90(24):11473-7.

68. McMahon-Pratt D, Rodriguez D, Rodriguez JR, Zhang Y, Manson K, Beroman C, et al. Recombinant vaccinia viruses expressing GP46/M-2 protect against Leishmania infection. Infect Immun. 1993 Aug;61(8):3351-9.

69. Gonzalo RM, del Real G, Rodriguez JR, Rodriguez D, Heljasvaara R, Lucas P, et al. A heterologous primeboost regime using DNA and recombinant vaccinia virus expressing the Leishmania infantum P36/ LACK antigen protects BALB/c mice from cutaneous leishmaniasis. Vaccine. 2002 Jan;20(7-8):1226-31.
70. Grimaldi G Jr, Teva A, Porrozzi R, Pinto MA, Marchersky RS, Rocha MG, et al. Clinical and parasitological protection in a Leishmania infantum-macaque model vaccinated with adenovirus and the recombinant A2 antigen. PLoS NegI Trop Dis. 2014 Jun;8(6):e2853. DOI 10.1371/journal.pntd.0002853.

71. Streit JA, Recker TJ, DoneIson JE, Wilson ME. BCG expressing LCR1 of Leishmania chagasi induces protective immunity in susceptible mice. Exp Parasitol. 2000 Jan;94(1):33-41.

72. Ramírez JR, Gilchrist K, Robledo S, Sepúlveda JC, Moll H, Soldati D, et al. Attenuated Toxoplasma gondii ts-4 mutants engineered to express the Leishmania antigen KMP-11 elicit a specific immune response in BALB/c mice. Vaccine. 2001 Nov;20(3-4):455-61.

73. Breton M, Tremblay MJ, Ouellette M, Papadopoulou B. Live nonpathogenic parasitic vector as a candidate vaccine against visceral leishmaniasis. Infect Immun. 2005 Oct;73(10):6372-82.

74. Dumas C, Muyombwe A, Roy G, Matte C, Ouellette $M$, Olivier $M$, et al. Recombinant Leishmania major secreting biologically active granulocytemacrophage colony-stimulating factor survives poorly in macrophages in vitro and delays disease development in mice. Infect Immun. 2003 Nor;7 1(11):6499-509.

75. Datta S, Roy S, Manna M. Therapy with radioattenuated vaccine in experimental murine visceral leishmaniasis showed enhanced $\mathrm{T}$ cell and inducible nitric oxide synthase levels, suppressed tumor growth factor-beta production with higher expression of some signaling molecules. Braz J Infect Dis. 2015 Jan-Feb;19(1):36-42. DOI 10.1016/j. bjid.2014.10.009.

76. Xu D, Liew FY. Genetic vaccination against leishmaniasis. Vaccine. 1994 Dec;12(16):1534-6.

77. Modabber F. Leishmaniasis vaccines: past, present and future. Int J Antimicrob Agents. 2010 Nor;36 SuppI 1:S58-61. DOI 10.1016/j.ijantimicag.2010.06.024.

78. Ramirez L, Corvo L, Duarte MC, Chávez-Fumagalli MA, Valadares DG, Santos DM, et al. Cross-protective effect of a combined L5 plus L3 Leishmania major ribosomal protein based vaccine combined with a Th1 adjuvant in murine cutaneous and visceral leishmaniasis. Parasit Vectors. 2014 Jan;7:3. DOI 10.1186/1756-3305-7-3. 
79. Monnerat S, Martinez-Calvillo S, Worthey E, Myler PJ, Stuart KD, Fasel N. Genomic organization and gene expression in a chromosomal region of Leishmania major. Mol Biochem Parasitol. 2004 Apr;134(2):233-43.

80. Tabatabaie F, Mahdavi M, Faezi S, Dalimi A, Sharifi Z, Akhlaghi L, et al. Th1 Platform Immune Responses Against Leishmania major Induced by Thiol-Specific Antioxidant-Based DNA Vaccines. Jundishapur J Microbiol. 2014 Feb;7(2):e8974. DOI 10.5812/ jjm.8974.

81. Goto Y, Coler RN, Reed SG. Bioinformatic identification of tandem repeat antigens of the Leishmania donovani complex. Infect Immun. 2007 Feb; 75(2):846-51.

82. Coler RN, Goto Y, Bogatzki L, Raman V, Reed SG. Leish-111f, a recombinant polyprotein vaccine that protects against visceral Leishmaniasis by elicitation of CD4+ T cells. Infect Immun. 2007 Sep;75(9):4648-54.

83. Encke J, zu Putlitz J, Wands JR. DNA vaccines. Intervirology. 1999;42(2-3): 117-24.

84. Osorio EY, Travi BL, da Cruz AM, Saldarriaga OA, Medina AA, Melby PC. Growth factor and Th2 cytokine signaling pathways converge at STAT6 to promote arginase expression in progressive experimental visceral leishmaniasis. PLoS Pathog. 2014 Jun;10(6):e1004165. DOI 10.1371/journal. ppat. 1004165 .

85. Griewank KG, Lorenz B, Fischer MR, Boon L, Lopez Kostka S, von Stebut E. Immune modulating effects of NKT cells in a physiologically low dose Leishmania major infection model after aGalCer analog PBS57 stimulation. PLoS Negl Trop Dis. 2014 Jun;8(6):e2917. DOI 10.1371/journal.pntd.0002917.

86. Moll H, Berberich C. Dendritic cell-based vaccination strategies: induction of protective immunity against leishmaniasis. Immunobiology. 2001 Dec;204(5):659-66.

87. Inaba K, Turley S, Iyoda T, Yamaide F, Shimoyama S, Reis e Sousa $\mathrm{C}$, et al. The formation of immunogenic major histocompatibility complex class II-peptide ligands in Iysosomal compartments of dendritic cells is regulated by inflammatory stimuli. J Exp Med. 2000 Mar;191(6):927-36.
88. Pulendran B, Palucka K, Banchereau J. Sensing pathogens and tuning immune responses. Science. 2001 Jul;293(5528):253-6.

89. Steinman RM. Lasker Basic Medical Research Award. Dendritic cells: versatile controllers of the immune system. Nat Med. 2007 Oct;13(10):1155-9.

90. Steinman RM, Banchereau J. Taking dendritic cells into medicine. Nature. 2007 Sep;449(7161):419-26.

91. Ahuja SS, Reddick RL, Sato N, Montalbo E, Kostecki $\nabla$, Zhao W, et al. Dendritic cell (DC)-based anti-infective strategies: DCs engineered to secrete IL-12 are a potent vaccine in a murine model of an intracellular infection. J Immunol. 1999 Oct;163(7):3890-7.

92. Schnitzer JK, Berzel S, Fajardo-Moser M, Remer KA, Moll H. Fragments of antigen-loaded dendritic cells (DC) and DC-derived exosomes induce protective immunity against Leishmania major. Vaccine. 2010 Aug;28(36):5785-93. DOI 10.1016/j.vaccine.2010.06.077.

93. Tsagozis P, Karagouni E, Dotsika E. Dendritic cells pulsed with peptides of gp63 induce differential protection against experimental cutaneous leishmaniasis. Int J Immunopathol Pharmacol. 2004 Sep-Dec; $17(3): 343-52$.

94. Patronov A, Doytchinova I. T-cell epitope vaccine design by immunoinformatics. Open Biol. 2013 Jan 8;3(1):120139. DOI 10.1098/rsob.120139.

95. Chu RS, Targoni OS, Krieg AM, Lehmann PV, Harding CV. CpG oligodeoxynucleotides act as adjuvants that switch on T helper 1 (Th1) immunity. J Exp Med. 1997 Nov;186(10):1623-31.

96. Matos I, Mizenina O, Lubkin A, Steinman RM, Idoyaga J. Targeting Leishmania major Antigens to Dendritic Cells In Vivo Induces Protective Immunity. PLoS One. 2013 Jun;8(6):e67453.

97. Tu H, Burke TM, Oderup C, Huang K, Wong K, Lewén $S$, et al. Robust expansion of dendritic cells in vivo by hydrodynamic FLT3L-FC gene transfer. J Immunol Methods. 2014 Nov;413:69-73. DOI 10.1016/j. jim.2014.07.008.

98. Kioy DW, Murilla G, Kofi-Tsekpo MW, Mukhongo M, Okwara J. Anti-trypanosomal effects of some compounds isolated from the extracts of Warburgia ugandensis. Afr J Health Sci. 1998 Feb;5(1):35-7. 
99. Brodie TM, Smith MC, Morris RV, Titus RG. Immunomodulatory effects of the Lutzomyia longipalpis salivary gland protein maxadilan on mouse macrophages. Infect Immun. 2007 May;75(5):2359-65.

100. Tavares NM, Silva RA, Costa DJ, Pitombo MA, Fukutani KF, Miranda JC, et al. Lutzomyia longipalpis saliva or salivary protein LJM19 protects against Leishmania braziliensis and the saliva of its vector, Lutzomyia intermedia. PLoS Negl Trop Dis. 2011;5(5):e1169. DOI 10.1371/journal.pntd.0001169.

101. Zahedifard F, Gholami E, Taheri T, Taslimi Y, Doustdari F, Seyed N, et al. Enhanced protective efficacy of nonpathogenic recombinant leishmania tarentolae expressing cysteine proteinases combined with a sand fly salivary antigen. PLoS Negl Trop Dis. 2014 Mar;8(3):e2751. DOI 10.1371/journal.pntd.0002751. 